\title{
Erratum to: Rapid generation of genetic diversity by multiplex CRISPR/Cas9 genome editing in rice
}

\author{
Lan Shen ${ }^{1,2 \dagger}$, Yufeng Hua ${ }^{1 \dagger}$, Yaping $\mathrm{Fu}^{1 \dagger}$, Jian $\mathrm{Li}^{3 \dagger}$, Qing Liu ${ }^{1}$, Xiaozhen Jiao ${ }^{1}$, Gaowei Xin ${ }^{4}$, \\ Junjie Wang ${ }^{1}$, Xingchun Wang ${ }^{4}$, Changjie Yan $^{2 *} \&$ Kejian Wang ${ }^{1 *}$ \\ ${ }^{1}$ State Key Laboratory of Rice Biology, China National Rice Research Institute, Chinese Academy of Agricultural Sciences, \\ Hangzhou 310006, China; \\ ${ }^{2}$ Key Laboratory of Plant Functional Genomics, Ministry of Education, Yangzhou University, Yangzhou 225009, China; \\ ${ }^{3}$ Lianyungang Academy of Agricultural Sciences, Lianyungang 222006, China; \\ ${ }^{4}$ College of Life Sciences, Shanxi Agricultural University, Taigu 030801, China
}

Erratum to: Science China Life Sciences, May 2017 Vol.60 No.5: 506-515, doi: 10.1007/s11427-017-9008-8

Citation: Shen, L., Hua, Y., Fu, Y., Li, J., Liu, Q., Jiao, X., Xin, G., Wang, J., Wang, X., Yan, C., et al. (2019). Erratum to: Rapid generation of genetic diversity by multiplex CRISPR/Cas9 genome editing in rice. Sci China Life Sci 62, 1270. https://doi.org/10.1007/s11427-019-9599-5

The same figure was misused for the PCR/RE assay results of Gnla and GW2 fragments in Figure 3, and the arrows in the graphicsal result of $G W 2$ were not on the tape. The corrected Figure 3 is as follows.

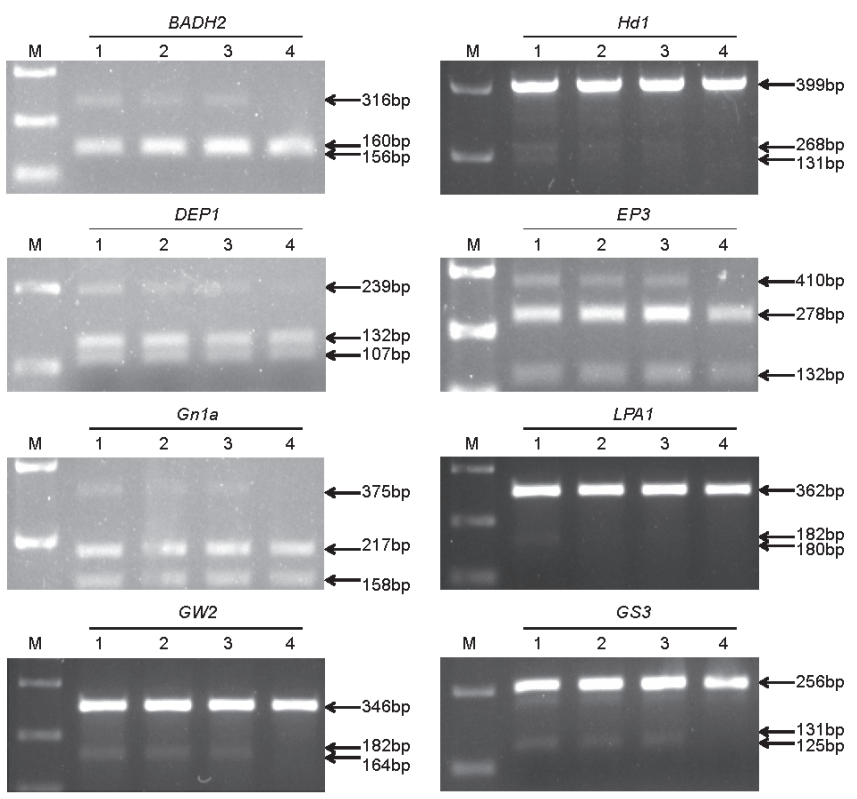

The online version of the original article can be found at https://link.springer.com/article/10.1007/s11427-017-9008-8

\footnotetext{
$\dagger$ Contributed equally to this work

*Corresponding authors (Changjie Yan, email: cjyan@yzu.edu.cn; Kejian Wang, email: wangkejian@ caas.cn)
} 